\title{
Corrosion inhibition of austenitic stainless steel by clay in polluted phosphoric acid with presence of SiC abrasif
}

\author{
S. Skal ${ }^{1}$, Y. Kerroum ${ }^{1}$, Y. El Aoufir ${ }^{1}$, A. Guenbour ${ }^{1}$, A. Bellaouchou ${ }^{1}$, H. Tabyaoui ${ }^{1}$ and H.Idrissi ${ }^{2}$ \\ ${ }^{1}$ Laboratoire des Matériaux Nanotechnologie et Environnement, Université Mohamed V Agdal. Faculté des \\ Sciences. Av. Ibn Battouta. BP 1014, Rabat. Maroc \\ ${ }^{2}$ Laboratoire des Matériaux Sciences et Ingénierie MATEIS, INSA Lyon, Bât. B. Pascal, $5^{\circ}$ étage - 7, Av. Jean \\ Capelle, 69621 Villeurbanne cedex. France
}

\begin{abstract}
Stainless steels have many properties mechanical and chemical resistances resulting from the formation of the protective layer (passive film) on their surface which prevents the metal to react with corrosive environments such as, phosphoric acid. This acid contains various impurities, including agressive agents and solid particles of gypsum, increase the risk of corrosion damage depending on the type of stainless steel used. In addition, it has been show that abrasion-corrosion causes an acceleration electrochemical process leading to a decrease in the resistance of materials. This work is to find a solution through an ecological inhibitor. That why we have been studied the effect of crude clay on corrosion behavior of Alloy 31 in polluted phosphoric acid with abrasive by electrochemical impedance spectroscopy (EIS) . The clay was characterized by X-ray fluorescence spectroscopy (FX), X-ray diffraction (DRX) and infrared spectroscopy (IR). EIS exhibited that resistance of Alloy 31 increased with increase the concentration of inhibitor.
\end{abstract}

\section{Introduction}

Le phénomène de corrosion-abrasion est habituellement causé par un corrodent aqueux ou gazeux qui coule sur la surface métallique ou qui l'empêche. La détérioration mécanique peut être aggravée par la présence d'un corrodent, comme dans le cas d'une usure fretante ou corrosive [1].

La corrosion-abrasion est affectée par de nombreux facteurs, à savoir, la vitesse, la turbulence, l'impact, la présence de solides en suspension, la température et les conditions de cavitation prédominantes [2,3]. L'accélération de l'attaque est due à la destruction ou à l'élimination du film protecteur par des forces mécaniques, exposant ainsi des surfaces métalliques fraîches anodiques au film voisin non ionisé. Un film dur, dense et adhérent, tel que sur l'acier inoxydable est plus résistant qu'un film fragile et mince, comme celui du plomb. La nature du film de protection dépend en grande partie de la corrosion elle-même [4].

La résistance à la corrosion des aciers inoxydables est déterminée par la formation d'un film protecteur à l'interface métal-solution. Cependant, cette passivité peut être profondément modifiée par l'action électrochimique, mécanique ou physique. Spécifiquement sous l'action de l'effet d'abrasion qui peut conduire à une destruction mécanique partielle ou totale du film passif [5].

Pour la protection contre la corrosion-abrasion, il est possible d'agir sur le matériau lui-même, sur la surface du matériau ou par modification de l'environnement (inhibiteurs de corrosion). Les inhibiteurs de corrosion sont un moyen original pour contrôler la corrosion des métaux [6].

L'inhibiteur utilisé dans ce travail est l'argile. Cette dernière provient de la région de Fès. Les argiles sont écologiques, pas toxiques, et peu coûteuses. L'importance chimique des minéraux argileux est due à leur structure cristalline, à leur surface élevée, à leur capacité d'échange ionique et à de bonnes propriétés de gonflement dans certains cas [7,8]. Les minéraux argileux sont importants en raison de leur grande échelle d'application dans l'industrie de fabrication de l'acide phosphorique et de la possibilité de modifier leur structure en couches par intercalation [9].

Notre étude porte sur la caractérisation minéralogique afin de l'utiliser comme inhibiteur dans l'acide phosphorique pollué $\left(\begin{array}{lll}40 \% & \mathrm{H}_{3} \mathrm{PO}_{4}\end{array}\right)$ 
dans le cas d'une corrosion-abrasion des aciers inoxydables.

\section{Matériel et méthodes}

\subsection{Matériaux et solutions testées}

L'acier inoxydable utilisé dans cette étude est l'acier allié austénitique (alliage 31). Sa composition chimique est donnée dans le tableau 1. La surface du matériau est de $0,5 \mathrm{~cm}^{2}$. Avant chaque essai, la surface de l'acier subit un polissage au papier abrasif de granulométrie croissante (de 180 à 1500). Ensuite, les spécimens sont lavés à l'eau distillée et séchés sous flux d'air.

La solution d'acide phosphorique étudiée est de $40 \%$ polluée par addition de $4 \%$ de $\mathrm{H}_{2} \mathrm{SO}_{4}$ et de $0,42 \%$ de $\mathrm{KCl}$, des concentrations typiques pour l'industrie de l'acide phosphorique, en présence de $\mathrm{SiC}$ abrasif.

Les concentrations utilisées pour l'argile est de $0.5 \mathrm{~g} / 1,2 \mathrm{~g} / \mathrm{l}, 4 \mathrm{~g} / \mathrm{l}$ et $6 \mathrm{~g} / 1$.

Dans tous les tests, l'angle d'impact est de $90^{\circ}$ et la concentration des particules abrasives $\mathrm{SiC}$ est de 24 $\mathrm{g} / 1$.

Tableau 1: Composition chimique de l'acier inoxydable austénitique en alliage 31 .

\begin{tabular}{|c|c|}
\hline \multicolumn{2}{|c|}{ Alliage 31 } \\
\hline Eléments & \% massique \\
\hline $\mathrm{Cr}$ & 26,75 \\
\hline $\mathrm{Fe}$ & 31,43 \\
\hline $\mathrm{Ni}$ & 31,85 \\
\hline $\mathrm{Mo}$ & 6,6 \\
\hline $\mathrm{Mn}$ & 1,5 \\
\hline $\mathrm{Cu}$ & 1,21 \\
\hline $\mathrm{N}$ & 0,193 \\
\hline $\mathrm{Si}$ & 0,1 \\
\hline $\mathrm{C}$ & 0,005 \\
\hline $\mathrm{S}$ & 0,002 \\
\hline $\mathrm{P}$ & 0,017 \\
\hline
\end{tabular}

\subsection{Mesures électrochimiques}

Les expériences électrochimiques ont été effectuées en utilisant un potentiostat Voltalab PGZ 301. La cellule électrochimique consiste en une électrode de travail (alliage 31), une électrode de platine en tant qu'électrode auxiliaire et une électrode au calomel saturé (ECS) comme électrode de référence. Avant les mesures électrochimiques, l'électrode de travail a été initialement réduite potentiostatiquement à $600 \mathrm{mV} / \mathrm{ECS}$ pendant $60 \mathrm{~s}$ afin d'éliminer les oxydes formés à l'air. Ensuite, l'électrode de travail a été immergée dans une solution d'essai pendant 2 $\mathrm{h}$ jusqu'à ce que le potentiel de circuit ouvert stable soit atteint. La spectroscopie d'impédance électrochimique a été effectuée dans la gamme de fréquences de $100 \mathrm{kHz}$ à $100 \mathrm{mHz}$, avec un signal d'amplitude de $10 \mathrm{mV}$.

\section{Résultats et discussions}

\subsection{Caractéristiques de l'inhibiteur}

\subsubsection{Fluorescence des rayons $X$ :}

La méthode d'analyse par fluorescence des rayons $\mathrm{X}$ est connu par sa rapidité, sa simplicité de mise en œuvre, sa faculté de doser plusieurs éléments sur la même préparation et la grande universalité des éléments dosables [10].

Le tableau 2 résume les différents composants chimiques de l'argile minérale provenant de la région de Fés. D'après le tableau, on remarque qu'il y a trois éléments majoritaires : la silice, le calcium et l'aluminium.

Tableau 2: Composition chimique en éléments majeurs d'argile minérale (Région Fés)

\begin{tabular}{|c|c|}
\hline Eléments & \% massique \\
\hline $\mathrm{SiO}_{2}$ & 38,86 \\
\hline $\mathrm{Al}_{2} \mathrm{O}_{3}$ & 10,76 \\
\hline $\mathrm{CaO}$ & 16,48 \\
\hline $\mathrm{Fe}_{2} \mathrm{O}_{3}$ & 4,42 \\
\hline $\mathrm{MgO}$ & 3,76 \\
\hline $\mathrm{K}_{2} \mathrm{O}$ & 1,62 \\
\hline $\mathrm{SO}_{3}$ & 0,31 \\
\hline $\mathrm{TiO}_{2}$ & 0,55 \\
\hline $\mathrm{MnO}_{2} \mathrm{P}_{5}$ & 0,04 \\
\hline $\mathrm{Na}_{2} \mathrm{O}$ & 0,18 \\
\hline $\mathrm{SrO}$ & 0,017 \\
\hline & 0,049 \\
\hline
\end{tabular}

Les résultats obtenus montrent une présence de kaolinite en raison du pourcentage très importante de la silice et de l'aluminium. Aussi, le pourcentage massique de calcium est très élevé, d'où la richesse de cette argile en calcite. La présence des oxydes $\mathrm{Fe}_{2} \mathrm{O}_{3}, \mathrm{MgO}, \mathrm{K}_{2} \mathrm{O}$ et $\mathrm{Na}_{2} \mathrm{O}$ montre que l'argile contient des impuretés.

\subsubsection{Diffraction des rayons $X$}

Le diagramme d'analyse par la diffraction des rayons $\mathrm{X}(\mathrm{DRX})$ est illustré dans la figure 1 :

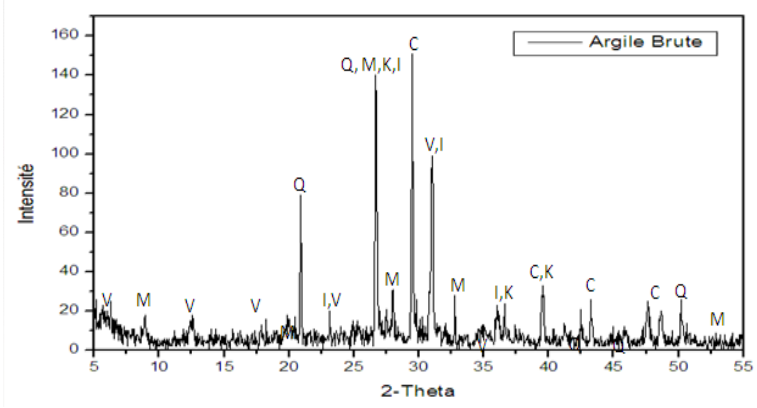

Fig 1. Spectre de la diffraction des rayons $\mathrm{X}$ de 1'argile brute 
L'analyse par DRX révèle que cette argile est composée de quartz $\left(\mathrm{SiO}_{2}\right)$, calcite $\mathrm{Ca}\left(\mathrm{CO}_{3}\right)$, vermiculite $\left[\mathrm{Mg}_{11} \mathrm{Al}_{5} \mathrm{FeSi}_{11} \mathrm{O}_{42,4} \mathrm{H}_{2} \mathrm{O}\right]$, muscovite $\left[\left(\mathrm{K}_{0.93} \mathrm{Na}_{0.07} \mathrm{Al}_{1.66} \mathrm{Fe}_{0.18} \mathrm{Mg}_{0.16}\left(\mathrm{Al}_{0.82} \mathrm{Si}_{3.18} \mathrm{O}_{10}\right)(\mathrm{OH})_{2}\right]\right.$, illite $\left[\left(\mathrm{K}, \mathrm{H}_{3} \mathrm{O}\right) \mathrm{Al}_{2} \mathrm{Si}_{3} \mathrm{AlO}_{10}(\mathrm{OH})_{2}\right]$ et kaolinite $\left(\mathrm{Al}_{2} \mathrm{Si}_{2} \mathrm{O}_{5}(\mathrm{OH})_{4}\right)$ [11].

Dans ce spectre (figure 1), nous remarquons une présence de deux pics intenses, l'un correspond à la présence d'un mélange de Quartz et de Vermiculite et l'autre correspond à la présence de Calcite. Ces résultats montrent que cette argile est une argile hétérogène. Cela confirme les résultats obtenus de l'analyse par fluorescence des rayons X.

\subsubsection{Spectroscopie d'infrarouge (IR)}

La structure chimique de l'argile étudiée a été identifiée en utilisant la technique IR. Le spectre obtenu est illustré par la figure 2 .

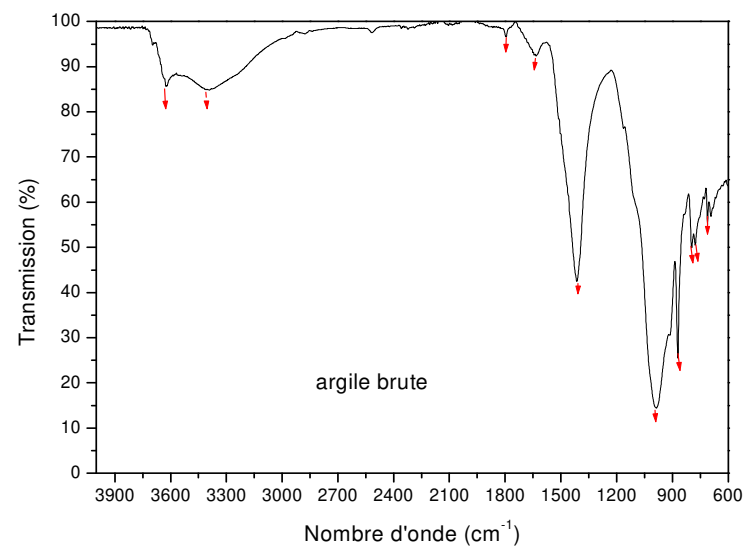

Fig 2. Spectre d'infrarouge de l'argile brute. On obtient :

- entre $3400 \mathrm{~cm}^{-1}$ et $3600 \mathrm{~cm}^{-1}$ : vibrations d'élongations du groupement $\mathrm{OH}$;

- $1800 \mathrm{~cm}^{-1}$ : vibrations d'élongations du groupement $\mathrm{C}=\mathrm{O}$;

- $1680 \mathrm{~cm}^{-1}$ : vibrations de valences $\mathrm{du}$ groupement $\mathrm{OH}$, due à l'eau de constitution de l'argile ;

- $1430 \mathrm{~cm}^{-1}$ : vibrations de déformation du groupe $\mathrm{CH}_{3}$;

- $1000 \mathrm{~cm}^{-1}$ : vibrations d'allongement de $\mathrm{Si}-\mathrm{O}$ (kaolinite) ;

- $\quad 880 \mathrm{~cm}^{-1}$ : présence de liaison C-O carbonate de calcium $\left(\mathrm{CaCO}_{3}\right)$;

- $\quad 791 \mathrm{~cm}^{-1}$ : liaisons Si-O-Al ;

- $780 \mathrm{~cm}^{-1}$ : quartz ;

- $712 \mathrm{~cm}^{-1}$ : liaison $\mathrm{C}-\mathrm{O}$ carbonate de calcium $\left(\mathrm{CaCO}_{3}\right)[12,13]$.

Ces résultats confirment ceux trouvés dans l'analyse par diffraction de rayon $X$ et cela par la présence de Quartz, Kaolinite et Illite.

Après ce traitement caractéristique, nous avons étudié l'effet inhibiteur pour de cette argile sur le comportement de l'alliage 31 dans le cas de la corrosion-abrasion.

\subsection{Efficacité de l'inhibition}

\subsubsection{Spectroscopie électrochimique (EIS)}

d'impédance

Pour compléter et comparer les résultats obtenus précédemment, le comportement à la corrosion de l'alliage 31, en solution d'acide phosphorique pollué, avec et sans inhibiteur a été étudié par spectroscopie d'impédance électrochimique (EIS) après $1 \mathrm{~h}$ d'immersion (Figure 4 ).

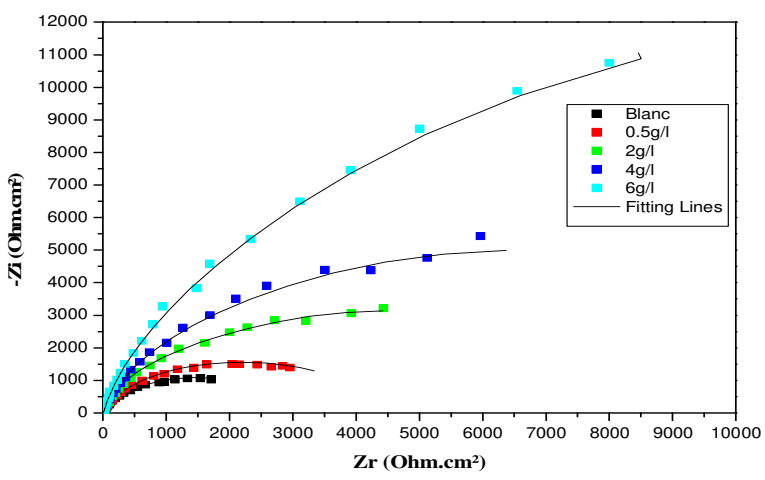

Fig 3. Les courbes de Nyquist de l'alliage 31 dans $40 \% \quad \mathrm{H}_{3} \mathrm{PO}_{4}$ pollué à différentes concentrations d'argile.

Les courbes de Nyquist ont été ajustées à l'aide des schémas électriques équivalents donnés sur la figure 5. Ce circuit se compose de la capacité et la résistance de la double couche $\left(\mathrm{C}_{1}, \mathrm{R}_{1}\right)$ placé en parallèle avec la capacité et la résistance du film passif $\left(\mathrm{C}_{2}, \mathrm{R}_{2}\right)$. Le symbole CPE (élément de phase constante) est utilisé pour signifier la possibilité d'une capacité non idéale attribuant à la distribution des temps de relaxation associés à des hétérogénéités à la surface de l'électrode.

Son impédance est définie par [14]:

$Z_{C P E}=\frac{1}{Q(j w)^{n}}$

$\mathrm{Q}$ est le module, $\omega$ et $\mathrm{n}$ sont la fréquence angulaire et la phase, respectivement et $\mathrm{j}^{2}=-1$ le nombre imaginaire. La capacité est considérée comme idéale lorsque $\mathrm{n}=1$, et non idéale lorsque $0,5<\mathrm{n}$ $<1$. Le CPE utilisé dans les circuits électriques équivalents de la figure 5 a été convertie en une capacité pure (C) au moyen de l'équation suivante [15] :

$C=\frac{(Q R)^{1 / n}}{R}$

Les principaux paramètres $\left(\mathrm{R}_{\mathrm{f}}, \mathrm{R}_{\mathrm{dl}}, \mathrm{C}_{\mathrm{f}}\right.$ et $\left.\mathrm{C}_{\mathrm{dl}}\right)$ extraits du diagramme de Nyquist sont regroupés dans le tableau 3. La résistance de polarisation est calculée par addition de la résistance du film et la résistance 
de la double couche: $R_{p}=R_{f}+R_{d l}$. Ainsi l'efficacité de l'inhibition est calculée par l'équation suivante [16]:

$\mathrm{EI}(\%)=\frac{R_{p}-R_{p}^{\circ}}{R_{p}} \times 100$

Où $R_{p}^{\circ}$ et $R_{p}$ représentent respectivement les résistances de polarisation sans et avec inhibiteur.

Table 3 : Les paramètres d'impédance de l'alliage 31 en milieu d'acide phosphorique pollué en absence et en présence de différentes concentrations d'argile:

\begin{tabular}{|c|c|c|c|c|c|}
\hline Milieu & Blanc & \multicolumn{4}{|c|}{ Argile } \\
\hline $\begin{array}{l}\text { Concentra } \\
\text { tion en } \\
\text { inhibiteur }\end{array}$ & ----- & $\begin{array}{l}0.5 \\
\mathrm{~g} / 1\end{array}$ & $\begin{array}{l}2 \\
g / 1\end{array}$ & $\begin{array}{l}4 \\
g / 1\end{array}$ & $\begin{array}{l}6 \\
g / 1\end{array}$ \\
\hline $\begin{array}{l}\mathrm{R}_{2} \\
\left(\Omega . \mathrm{cm}^{2}\right)\end{array}$ & 522 & 857 & 998 & 1587 & $\begin{array}{l}392 \\
1\end{array}$ \\
\hline $\begin{array}{l}\mathrm{C}_{2} \\
\left(10^{-}\right. \\
\left.{ }^{4} \mathrm{~F} / \mathrm{cm}^{2}\right)\end{array}$ & 3,56 & 2,96 & 2,88 & 2,6 & 1,67 \\
\hline $\begin{array}{l}\mathrm{R}_{1} \\
\left(\Omega . \mathrm{cm}^{2}\right)\end{array}$ & 2291 & 3604 & $\begin{array}{l}751 \\
6 \\
\end{array}$ & 11162 & $\begin{array}{l}231 \\
30 \\
\end{array}$ \\
\hline $\begin{array}{l}\mathrm{C}_{1} \\
\left(10^{-}\right. \\
\left.{ }^{4} \mathrm{~F} / \mathrm{cm}^{2}\right) \\
\end{array}$ & 2.54 & 2,17 & 2,38 & 1,65 & 1,25 \\
\hline $\begin{array}{l}\mathrm{R}_{\mathrm{P}} \\
\left(\Omega . \mathrm{cm}^{2}\right)\end{array}$ & 2813 & 4461 & $\begin{array}{l}851 \\
4 \\
\end{array}$ & 12749 & $\begin{array}{l}27 \\
051 \\
\end{array}$ \\
\hline EI (\%) & ----- & 37 & 67 & 78 & 88 \\
\hline
\end{tabular}

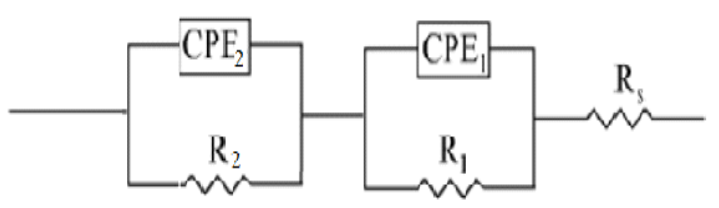

Figure 5. Le modèle de circuit équivalent de l'EIS.

A partir des résultats du tableau 4 on voit bien que l'augmentation de la concentration en inhibiteur engendre un accroissement des valeurs de $R_{p}$ ainsi qu'une diminution de la capacité de double couche. Cela indique que l'argile inhibe la corrosion-abrasion. La diminution des valeurs $\mathrm{C}_{\mathrm{dl}}$ en présence de l'argile peuvent être attribuer à la formation du film d'oxyde protecteur sur la surface du métal qui force la zone de passivation en présence de l'abrasif.

$\mathrm{M}+\mathrm{H}_{2} \mathrm{O} \rightarrow \mathrm{MO}+2 \mathrm{H}^{+}+2 \mathrm{e}-$

$2 \mathrm{MO}+\mathrm{H}_{2} \mathrm{O} \rightarrow \mathrm{M}_{2} \mathrm{O}_{3}+2 \mathrm{H}^{+}+2 \mathrm{e}-$

Avec M soit le Fe, Cr ou Ni. [17]

Ceci est confirmé par d'autres auteurs tels que M. Larouj et al., [18] qui a travaillé sur l'effet inhibiteur de AOPT sur le comportement de l'acier au carbone dans une solution de $2 \mathrm{M} \mathrm{H}_{3} \mathrm{PO}_{4}$. Il a conclu que le diamètre de la boucle capacitive augmente avec l'augmentation de la concentration en AOPT, suggérant que le film inhibiteur formé a été renforcé par l'ajout de AOPT. Ainsi M. Boudalia et al., [16] a montré que l'argile s'adsorbe sur la surface du métal et forme un film qui réduit la surface métallique subissant la corrosion.

\section{Conclusion}

Les techniques de caractérisation de l'argile provenant de la région de Fès ont révélé qu'elle est hétérogène et qu'elle est composée d'un mélange de quartz, muscovite, illite et kaolinite et de calcite.

Les études de polarisation ont montré que l'argile est un inhibiteur de type anodique dans les conditions de corrosion-abrasion qui diminue la dissolution anodique de métal et que son efficacité de corrosion augmente avec la concentration d'inhibiteur. Les résultats d'impédance ont montré que la résistance de la polarisation augmente avec l'accroissement de la concentration en argile. Une meilleure performance d'environ $88 \%$ est observée en présence de $6 \mathrm{~g} / \mathrm{L}$ de l'inhibiteur.

\section{References}

1. S.S. Rajahram, T.J. Harvey, R.J.K. Wood, Wear 267 244-254(2009).

2. F.Mohammadi, J.Luo, Corros.Sci 52, 2994 3001(2010).

3. M. Lindgren, S. Siljander, R. Suihkonen, P. Pohjanne, J. Vuorinen, Wear, 364-365 (2016).

4. A. Guenbour, M.A. Hajji, E.M. Jallouli, A. Ben Bachir, Appl. Surf. Sci. 253, 2362-2366 (2006).

5. S. Skal, Y. Kerroum, A. Guenbour, A. Bellaouchou, H. Tabyaoui, H. Idrissi, A. Zarrouk, J. García-Antón, J. Mater. Environ. Sci. 8, 3234-3246 (2017).

6. Y. EL Aoufir, H. Lgaz, H. Bourazmi, Y. Kerroum, Y. Ramli, A. Guenbour, R. Salghi, F. ElHajjaji, B. Hammouti, H. Oudda, J. Mater. Environ. Sci. 7, 4330-4347 (2016).

7. L. Jancovic, P. Komadel, J. Catal. 218, 227 (2003).

8. M. Rouli, A.A. Vassiliadis. J. Colloid Interf. Sci. 291, 37-44 (2005).

9. D.I. Nistor, N.D. Miron, I. Siminiceanu, Scientific Study \& Research 6, (2006).

10. C. Sadik, I.EL Amrani EL Hassani, A. ALbizane, , INVACO2.

11. S. Louati, S. Baklouti, B. Samet, Hindawi Publishing Corporation Advances in Materials Science and Engineering (2016).

12. A. Qlihaa, S. Dhimni, F. Melrhaka, N. Hajjaji, A. Srhiri,, J. Mater. Environ. Sci. 7, 1741-1750 (2016).

13. O.Qabaqous, N.Tijani, M.Naciri Bennani, A.El Krouk, J. Mater. Environ. Sci. 5, 2247 (2014).

14. FB.Growcock, R.J.Jasinski, J.Electrochem. Soc. 136,(1989).

15. C. Escrivà-Cerdán, E. Blasco-Tamarit, D.M. García-García, J. García-Antón, R. Akid, J. Walton, Electrochimica Acta 111, 552- 561 (2013).

16. M. Boudalia, A. Guenbour, A. Bellaouchou, A. Zarrouk, Der Pharma Chemica 7, 301-306 (2015). 
17. H. Iken, R. Basseguy, A. Guenbour, A. Ben Bachir, Electrochimica Acta 52, 2580-2587 (2007). 18. M. Larouj, Y. EL Aoufir, H. Serrar, A. El Assyry, M. Galai, A. Zarrouk, B. Hammouti, A.
Guenbour, A. El Midaoui, S. Boukhriss, M. Ebn Touhami, H. Oudda, Der Pharmacia Lettre 6, 324334 (2014). 